\title{
Downregulation of PDCD4 by miR-21 suppresses tumor transformation and proliferation in a nude mouse renal cancer model
}

\author{
HAIXIN YUAN $^{1 *}$, SHIYONG XIN ${ }^{1 *}$, YAOPING HUANG ${ }^{2}$, YINGFAN BAO $^{3}$, HAO JIANG $^{4}$, \\ LIQING ZHOU $^{5}$, XIAOQIANG REN ${ }^{1}$, LIANG LI ${ }^{1}$, QIAN WANG ${ }^{1}$ and JIANGUO ZHANG ${ }^{1}$
}

\begin{abstract}
${ }^{1}$ Department of Urology, The First Affiliated Hospital, College of Clinical Medicine of Henan University of Science and Technology, Luoyang, Henan 471003; ${ }^{2}$ Medical School of Pingdingshan University, Pingdingshan, Henan 467000; ${ }^{3}$ Zhengzhou Shuqing Medical College, Zhengzhou, Henan 450000; ${ }^{4}$ Department of General Surgery, Wendeng Central Hospital of Weihai, Weihai, Shandong 264200; ${ }^{5}$ Department of Rheumatism Immunity, The First Affiliated Hospital, College of Clinical Medicine of Henan University of Science and Technology, Luoyang, Henan 471003, P.R. China
\end{abstract}

Received September 5, 2015; Accepted March 7, 2017

DOI: $10.3892 / 01.2017 .6605$

\begin{abstract}
Programmed cell death 4 (PDCD4) is known to suppress neoplastic transformation, cell proliferation and metastasis, and to be downregulated by microRNA-21 (miR-21) in renal cell carcinoma (RCC) cell lines and tissues. The aim of the present study was to investigate the roles of and association between PDCD4 and miR-21 in a nude mouse renal cancer model. A total of $24 \mathrm{BALB} / \mathrm{c}$ male nude mice were randomly assigned into the following three groups: Negative control $(\mathrm{NC} ; \mathrm{n}=8)$, miR-21 inhibitor $(\mathrm{n}=8)$ and miR-21 mimic $(n=8)$. Subsequently, renal cell adenocarcinoma 786-O cells were subcutaneously transplanted into the armpits of the mice, which were then injected daily with NC small interfering (si) RNA, precursor-miR-21 (mimic) or anti-miR-21 (inhibitor). Tumors were removed from the mice and weighed 16 days following 786-O cell transplantation. In addition, the expression of miR-21 and PDCD4 mRNA in cancer tissues was analyzed using reverse transcription-quantitative PCR. The expression of PDCD4 protein in cancer tissues was also examined using immunohistochemistry and western blotting. Furthermore, 786-O cells were transfected with PDCD4 siRNA or NC siRNA, and the effects of silencing PDCD4 on tumor cell
\end{abstract}

Correspondence to: Professor Jianguo Zhang or Mr. Shiyong Xin, Department of Urology, The First Affiliated Hospital, College of Clinical Medicine of Henan University of Science and Technology, 24 Jinghua Road, Luoyang, Henan 471003, P.R. China

E-mail: doctjgzhang@sina.com

E-mail: xinshiyong66@163.com

*Contributed equally

Key words: programmed cell death 4, microRNA-21, nude mice, renal cancer growth, proliferation and invasion were investigated using soft agar colony formation, EdU cell proliferation assay and Transwell migration and invasion assays. Another 16 BALB/c male nude mice were randomly assigned into two groups as follows: NC $(n=8)$ and PDCD4 siRNA $(n=8)$. The 786-O cells were subcutaneously transplanted into the armpits of the mice, which were subsequently injected daily with NC siRNA or PDCD4 siRNA. The tumors were removed and weighed 16 days following transplantation. Compared with the NC group, tumor weight in the miR-21 mimic group was significantly increased. By contrast, tumor weight in the miR-21 inhibitor group was significantly decreased. Similar to the results observed in human renal cancer tissue and cell lines, miR-21 expression in the nude mouse renal cancer models was significantly upregulated in the miR-21 mimic group compared with the NC group, while it was significantly lower in the miR-21 inhibitor group. Furthermore, there was a significant reduction in PDCD4 protein levels in the miR-21 mimic group and a significant increase in the miR-21 inhibitor group compared with the NC, whereas PDCD4 mRNA expression was not significantly altered. In the EdU proliferation assay, the mean percentage of new cells that incorporated EdU was $28.6 \%$ in the NC siRNA group and significantly increased to $44.7 \%$ in PDCD4 siRNA transfected cells. In the soft agar colony formation assay, Transwell and migration and invasion assays, a significant increase in colony formation, migration and invasion capacity in PDCD4 siRNA-transfected cells was observed compared with the NC. Furthermore, compared with the NC group, tumor weight in the PDCD4 siRNA group was significantly increased. Similar to the results observed in human renal cancer tissue and cell lines, miR-21 promoted cancer cell hyperplasia and proliferation, and post-transcriptionally downregulated PDCD4 protein expression, in the nude mouse renal cancer model. The results of the present study and previous studies indicate that PDCD4 and miR-21 serve an important role in renal cancer. Thus, increasing PDCD4 expression or inhibiting miR-21 expression may constitute effective novel therapeutic strategies for the treatment of renal cancer. 


\section{Introduction}

Renal cell carcinoma (RCC) is the third most common urological cancer (1). A number of patients with RCC develop metastatic disease and the 5-year survival rate in these patients just $2 \%$ (2). Therefore, further screening and investigations into novel treatment methods are highly warranted.

MicroRNAs (miRNAs/miRs) are non-coding small RNAs of 19-25 nt in length which are cleaved from 70-100 nt-long hairpin precursor (pre)-miRNAs by the enzyme ribonuclease 3 (Drosha) $(3,4)$. miRNAs have essential functions in the development and establishment of cell identity, and aberrant metabolism or expression of miRNAs has been associated with human disease, including cancer (4). There are an increasing number of reports implicating aberrant expression of certain miRNAs, including miR-21, 17-92,-15,-16,-141 and let-7, in tumor growth, carcinogenesis and response to chemotherapy in various malignancies (3-10). miR-21, which is overexpressed in various cancer types, is one of the most widely studied miRNAs in cancer (11-13). The overexpression of miR-21 has been implicated in various processes associated with carcinogenesis, including the inhibition of apoptosis (6), promotion of cell proliferation (9) and stimulation of tumor growth (10).

Programmed cell death 4 (PDCD4) has been demonstrated to be an inhibitor of neoplastic transformation. The PDCD4 gene was identified in the mouse epidermal clonal genetic variant JB6 cell system as a $64 \mathrm{kDa}$ protein that is preferentially expressed in tumor promoter-resistant cells, but suppressed in tumor promoter-sensitive cells undergoing neoplastic transformation (14). PDCD4 levels were continuously reduced in the colon and colorectal adenocarcinoma $(15,16)$. PDCD4 inhibits activator protein (AP)-1 transactivation (17), stalls translation machinery (18), decreases benign and malignant tumor progression (19), and regulates lymphoma initiation and autoimmune inflammation (20). Subsequent investigations demonstrated that a loss of PDCD4 expression was associated with tumor progression in carcinomas of the lung, colon, prostate and breast (21).

Previous bioinformatics analyses have demonstrated that PDCD4 contains a miR-21 binding site and acts as a tumor suppressor through the regulation of various processes associated with cancer progression, including cell proliferation, invasion, metastasis and neoplastic transformation (21-23). Notably, Asangani et al (11) studied 10 colorectal cell lines and observed an inverse correlation between miR-21 and PDCD4 protein expression. Lu et al (24) demonstrated that translation of the tumor suppressor gene, PDCD4, is negatively regulated by miR-21 in HEK-293T, MCF-7 and JB6 cell lines, and provided evidence that the miR-21 gene functions as an oncogene to promote cell transformation. In human hepatocellular carcinoma cell lines, overexpression of miR-21 did not cause degradation of PDCD4 mRNA, but significantly inhibited its protein expression (25). It has been demonstrated that knockdown of miR-21 upregulates PDCD4 expression leading to increased apoptotic cell death in glioblastoma cells (26), in addition to suppressing invasion and metastasis in colorectal cancer cells (22) and esophageal squamous cell carcinoma (27). Further studies have confirmed the regulation of PDCD4 by miR-21 in colon, breast and bladder carcinoma (22), cholangiocarcinoma (23), esophageal carcinoma (27) and glioblastoma (26). Consistent with these results, Li et al (28) demonstrated that miR-21 is significantly overexpressed in RCC tissue and cell lines, and that PDCD4 is negatively regulated by miR-21.

To the best of our knowledge, no previous studies have elucidated the roles of and associations between miR-21 and PDCD4 in an animal RCC model. Therefore, the aim of the present study was to determine the roles of and interactions between PDCD4 and miR-21 in a nude mouse renal cancer model, and the effects of silencing PDCD4 on RCC tumor cell growth and invasion.

\section{Materials and methods}

Ethics statement. The present study was performed in strict accordance with the recommendations of the Guide for the Care and Use of Laboratory Animals of the National Institutes of Health (29). The protocol used in the present study was approved by the Committee on the Ethics of Animal Experiments at Henan University of Science and Technology (Henan, China; approval no. 20140126). All surgical procedures were performed under $1 \%$ sodium pentobarbital anesthesia $70 \mathrm{mg} / \mathrm{kg}$, and all efforts were made to minimize suffering. As a humane endpoint, mice were euthanized if they met any of the following conditions: i) When they exhibited loss of $>20 \%$ of body weight; ii) when the tumor mass $>10 \%$ of body weight; iii) when an increased respiratory rate and/or effort was observed; iv) if a loss of skin elasticity was observed; v) if the mice exhibited the inability to access food or water. Mice were injected with an excess of sodium pentobarbital anesthetic $(150 \mathrm{mg} / \mathrm{kg}, 1 \%)$ as the method of euthanasia.

Rearing of nude mice and cell culture. BALB/c nude mice $(\mathrm{n}=24$; male) were obtained from the Laboratory Animal Center of the Academy of Military Medical Sciences (Shanghai, China). These BALB/c nude mice were 5-6 weeks, weight 20-23 g, and they were fed for 1 week prior to the experiment under the food condition of SPF at $20-26^{\circ} \mathrm{C}$, relative humidity $40-70 \%$ and $12 \mathrm{~h}$ light-dark cycle. All food was treated with high temperature steam disinfection (45 min, $120^{\circ} \mathrm{C}$ ). All water was acidified by hydrochloric acid and adjusted to a pH between 2.5 and 2.8. Renal cell adenocarcinoma 786-O cells were obtained from the Xiehe Cell Bank of the Chinese Academy of Medical Sciences (Beijing, China) and were cultured under the conditions recommended by the cell bank. Briefly, the 786-O cells were cultured as a monolayer in Keratinocyte Serum-Free medium (Invitrogen; Thermo Fisher Scientific, Inc., Waltham, MA, USA) supplemented with $0.05 \mathrm{mg} / \mathrm{ml}$ bovine pituitary extract (Invitrogen; Thermo Fisher Scientific, Inc.), $5 \mathrm{ng} / \mathrm{ml}$ human recombinant epidermal growth factor (Invitrogen; Thermo Fisher Scientific, Inc.) and $10 \%$ fetal bovine serum (FBS; Atlanta Biologicals Inc., Lawrenceville, GA, USA), $50 \mathrm{mg} / \mathrm{ml}$ penicillin and $50 \mathrm{mg} / \mathrm{ml}$ streptomycin (both Invitrogen; Thermo Fisher Scientific, Inc.). Cells were maintained in an incubator with a humidified atmosphere and $5 \% \mathrm{CO}_{2}$ at $37^{\circ} \mathrm{C}$. Subconfluent $786-\mathrm{O}$ cells (60-70\% confluence) were treated with genistein $(25 \mathrm{mM}$; Sigma-Aldrich; Merck KGaA, Darmstadt, Germany) and $10 \%$ dimethyl sulfoxide $(15 \mathrm{ml})$. 
RNA interference assay. The 786-O cells ( $1 \times 10^{6}$ cells, $60-80 \%$ confluence) were incubated in a 6 -well tissue culture dish without antibiotics for $24 \mathrm{~h}$ prior to transfection. The 786-O cells were transfected with PDCD4 siRNA and the negative control siRNA, and the effects of silencing PDCD4 on tumor cell growth, proliferation and invasion were investigated. The small interfering RNA (siRNA) transfection reagent complexes (\#AM16708A; Invitrogen; Thermo Fisher Scientific, Inc.) were mixed with Lipofectamine ${ }^{\circledR} 2000$ (Invitrogen; Thermo Fisher Scientific, Inc.) according to the manufacturer's instructions and subsequently added to the cells. The sequences of the siRNAs used in the present study were as follows: NC siRNA (sense, 5'-GCUGCUUTGGACAAGGCUATC-3'; antisense, 5'-UAGCCUAGUCCAAAGCAGCAT-3') PDCD4 siRNA (sense, 5'-GCUGCUUUGGACAAGGCUATT-3'; antisense, 5'-UAGCCUUGUCCAAAGCAGCTT-3'). After $6 \mathrm{~h}$ of incubation at $37^{\circ} \mathrm{C}$, the medium was replaced and the cells were cultured in RPMI-1640 (Gibco; Thermo Fisher Scientific, Inc.) supplemented with $10 \%$ heat-inactivated FBS for various time periods. At the same time, cells were transfected with control siRNA as control group.

Nude mouse renal cancer model. The male nude mice were randomly assigned into the following three groups to investigate the effect of miR-21: Negative control ( $\mathrm{NC}$; $\mathrm{n}=8)$, miR-21 mimic $(n=8)$ and miR-21 inhibitor $(n=8)$. A $0.1 \mathrm{ml} 786-\mathrm{O}$ cell suspension $\left(1 \times 10^{6}\right.$ cells) was subcutaneously transplanted into the armpits of the mice, which were subsequently injected daily with NC siRNA (\#AM17110; Invitrogen; Thermo Fisher Scientific, Inc.), pre-miR-21 (mimic) or anti-miR-21 (inhibitor); (\#A25576; Invitrogen; Thermo Fisher Scientific, Inc.). The sequences of the primers as follows: pre-miR-21 primer sequence, sense, 5'-CATCCTUCUTGAAGUGAC UC-3' and antisense, 5'-CGCUCUAUGACGUAUGGA GGU-3'; anti-miR-21 primer sequence, sense, 5'-GATCCA UCUTCGAAGUGACTT-3' and antisense, 5'-UGCUCU TUGACGUAUGGAGTT-3'; NC siRNA primer sequence, sense, 5'-UUCACCGUACGUCUCACCUGT-3' and antisense, 5'-ACUGGAACCUCUCGCGGAATT-3'. MTT assays (Roche Diagnostics GmbH, Mannheim, Germany) were performed to detect cell viability. Cells were seeded into 96-well plates $\left(6.0 \times 10^{3}\right.$ cells/well) and left at normal culture conditions. Cells were incubated with MTT (5 mg/ml per well) for $4 \mathrm{~h}$. Dimethyl sulfoxide was used to dissolve the formazan crystals. PBS was used as a control. Absorbance at a wavelength of $490 \mathrm{~nm}$ was measured using the Infinite M200 PRO multimode microplate reader (Tecan Benelux BVBA, Mechelen, Belgium). Cell viability was detected prior to the injection of cells to ensure that the cells were in logarithmic phase. A total of 16 days after transplantation, the tumor formation rate was $100 \%$ and the mice were sacrificed, with the tumors collected and weighed. The expression of miR-21 and PDCD 4 mRNA in the cancer tissues was analyzed using reverse transcription-quantitative polymerase chain reaction (RT-qPCR).

The expression of PDCD4 protein in cancer tissues was examined using immunohistochemistry and western blotting. BALB/c mice were randomly assigned into two groups as follows: $\mathrm{NC}(n=8)$ and PDCD4 siRNA $(n=8)$. The 786-O cells were subcutaneously transplanted into the armpits of the mice, and this was followed by daily injections of NC
siRNA or PDCD4 siRNA (\#AM16708A; Invitrogen; Thermo Fisher Scientific, Inc.). NC siRNA sense, 5'-GCUGCUUTG GACAAGGCUATC-3' and antisense, 5'-UAGCCUAGUCCA AAGCAGCAT-3'; PDCD4 siRNA sense, 5'-GCUGCUUUG GACAAGGCUATT-3' and antisense, 5'-UAGCCUUGUCCA AAGCAGCTT-3'. The tumors were removed from the mice and weighed 16 days after the transplantation. Sodium pentobarbital $(150 \mathrm{mg} / \mathrm{kg}, 1 \%)$ was injected into each mouse as the mode of euthanisia.

Immunohistochemistry. For immunohistochemical analysis, mouse cancer tissues were fixed with $10 \%$ buffered formalin and embedded in paraffin. The sections $(2-\mu \mathrm{m}$-thick) were cut 1 day prior to use. All sections were deparaffinized and dehydrated with graded ethyl alcohol $(99,95,85$ and $75 \%)$. The sections were then washed for $10 \mathrm{~min}$ in phosphate-buffered saline (PBS; $\mathrm{pH} 7.2 ; 37^{\circ} \mathrm{C}$ ). The endogenous peroxidase activity was quenched by incubation in methanol containing $3 \% \mathrm{H}_{2} \mathrm{O}_{2}$ for $10 \mathrm{~min}$ at room temperature, then heated for $30 \mathrm{~min}$ at $95^{\circ} \mathrm{C}$ to repair antigens and finally washed with PBS. To maximize immunohistochemistry signals, the following two strategies were used: Antigen retrieval in citrate buffer and signal amplification with biotinylated tyramide. The tissue sections were incubated overnight at $4^{\circ} \mathrm{C}$ with PDCD4 antibody (dilution, 1:100; \#ab105998; Abcam, Cambridge, UK). Detection was subsequently performed using biotinylated goat anti-rabbit antibody (dilution, 1:5,000; \#SAB4504290; Sigma-Aldrich; Merck KGaA) and the tissue sections were incubated at $37^{\circ} \mathrm{C}$ for $20 \mathrm{~min}$. Diaminobenzidine was used as a chromogen, and the slide was counterstained with Mayer's hematoxylin. The results were independently observed under a fluorescent microscope (Olympus BX41; Olympus Corporation, Tokyo, Japan) at x400 magnification by two pathologists. For the NC, the primary antibody was replaced by $10 \%$ non-immune goat serum.

Western blot analysis. Western blot analyses were performed to determine the expression of PDCD4. Total protein was extracted from tissues using a Total Protein Extraction kit (Nanjing KeyGen Biotech Co., Ltd., Nanjing, China) according to the manufacturer's recommendations. The concentration of protein was measured using a BCA Assay kit (Nanjing KeyGen Biotech Co., Ltd.). The protein samples were separated on a $10 \%$ polyacrylamide gel using SDS-PAGE and transferred onto a hybond polyvinylidene difluoride membrane (GE Healthcare Bio-Sciences, Pittsburgh, PA, USA). The membranes were subsequently blocked in 5\% fat-free milk at room temperature for $2 \mathrm{~h}$. Following incubation with rabbit or goat primary antibodies directed against PDCD4 (1:10,000; cat no. ab80590; Abcam) or GAPDH (1:200; Santa Cruz Biotechnology, Inc., Dallas, TX, USA) at $4{ }^{\circ} \mathrm{C}$ overnight, the membranes were probed with goat anti-rabbit (\#SAB2502080; Sigma-Aldrich; Merck KGaA) or mouse anti-goat secondary antibodies (\#G8795; Sigma-Aldrich; Merck KGaA) at a dilution of 1:5,000 at room temperature for $2 \mathrm{~h}$. The signals were detected using a Super Enhanced Chemiluminescence Plus kit (Nanjing KeyGen Biotech Co., Ltd.) and quantified using UVP software (BioDoc-It ${ }^{\circledR}$ Imager System; UVP, LLC, Upland, CA, USA). The integrated optical density (IOD) ratio $\mathrm{IOD}_{\mathrm{PDCD} 4} / \mathrm{IOD}_{\mathrm{GAPDH}}$ was used to indicate the relative expression of PDCD4 protein at a wavelength of $280 \mathrm{~nm}$. 
$R T$ - $q P C R$. Total RNA was extracted from tumors using an RNA Isolation kit (CWbiotech Co., Ltd., Beijing, China) following the manufacturer's protocol. Stem-loop RT-qPCR for mature miR-21 was performed as previously described (28). RT-qPCR for PDCD4 was performed using Power SYBR ${ }^{\circledR}$ Green PCR Master mix (Agilent Technologies, Inc., Santa Clara, CA, USA) in a final volume of $20 \mu \mathrm{l}$, comprising of $100 \mathrm{ng}$ cDNA, $10 \mu \mathrm{l}$ master mix, $1 \mu \mathrm{l} \mathrm{ROX}$ and $0.4 \mathrm{pmol} / \mu \mathrm{l}$ of each primer. qPCR cycling conditions were as follows: $95^{\circ} \mathrm{C}$ for $2 \mathrm{~min}$, and then $95^{\circ} \mathrm{C}$ for $15 \mathrm{sec}$ and $55^{\circ} \mathrm{C}$ for $30 \mathrm{sec}$, for 40 cycles, followed by $60^{\circ} \mathrm{C}$ for $1 \mathrm{~min}$. The melting curve was $65-95^{\circ} \mathrm{C}$. Human U6 mRNA was used for normalization for the stem-loop RT-qPCR and GAPDH was used for normalization for the PDCD4 RT-qPCR. Fluorescent signals were normalized to these internal reference genes, and the threshold cycle (Cq) was set within the exponential phase of the PCR. The relative gene expression was calculated by comparing cycle times for each target PCR. The target PCR $\mathrm{Cq}$ values were normalized by subtracting the U6 or GADPH Cq value, which provided the $\Delta \mathrm{Cq}$ value. The relative expression level between treatments was then calculated using the following equation: Relative gene expression $=2^{(\Delta \text { Cqsample }-\Delta \text { Cqcontrol })}(30)$.

Primers. For miR-21 RT-qPCR, the primer sequence was 5-TAGCTTATCAGACTGATGTTGA-3, and reverse 5-AAC GCTTCACGAATTTGCGT-3. The other primer sequences for RT-qPCR were as follows: U6 forward, 5-CTCGCTTCG GCAGCACA-3 and reverse, 5-AACGCTTCACGAATTTGC GT-3; PDCD4 forward, 5-AGGCCGAGGTGGGCGGATCAC TTGA-3 and reverse, 5-GCCACCATGCCTGGCTACT-3; and GAPDH forward, 5-CCTCTGACTTCAACAGCGACAC-3 and reverse, 5-TGGTCCAGGGGTCTTACTCC-3.

EdU incorporation cell proliferation assay. Transfected 786-O cells were plated in 24-well plates at density of $4 \times 10^{4}$ cells/well, allowed to adhere for $5 \mathrm{~h}$, washed with PBS and then incubated in serum-free RPMI-1640 containing $10 \mu \mathrm{mol} / 1$ EdU (Guangzhou RiboBio Co., Ltd., Guangzhou, China) for $2 \mathrm{~h}$ at $37^{\circ} \mathrm{C}$. The cells were subsequently washed with PBS, and then fixed and permeabilized in PBS containing 2\% formaldehyde, $0.5 \%$ Triton $\mathrm{X}-100$, and $300 \mathrm{mmol} / 1$ sucrose for $15 \mathrm{~min}$ at $37^{\circ} \mathrm{C}$. Following washing with PBS, the cells were blocked using $10 \%$ FBS in PBS at ambient temperature for $30 \mathrm{~min}$, and incorporated EdU was detected by incubation with a fluorescent azide coupling solution (\#C10310-3; Apollo; Guangzhou RiboBio Co. Ltd) for $30 \mathrm{~min}$ at ambient temperature. The cells were washed three times with PBS containing $0.05 \%$ Tween-20, incubated with the DNA staining dye Hoechst 33342 for $30 \mathrm{~min}$ and washed in PBS. Images were captured using a fluorescent microscope, and the nuclear fluorescent intensity was calculated from $\geq 50$ non-S phase cells randomly selected in five different fields of view.

Soft agar colony formation assay. The bottom layer of the $15-\mathrm{cm}$ plate $(0.6 \%$ low-melt agarose) was prepared with RPMI-1640 medium containing 10\% FBS, $100 \mathrm{U} / \mathrm{ml}$ penicillin and $100 \mathrm{mg} / \mathrm{ml}$ streptomycin, in addition to $5 \times 10^{2}$ of the transfected 786-O cells. The top layer of the plate $(0.3 \%$ low-melt agarose) was prepared with RPMI 1640 medium containing $10 \% \mathrm{FBS}, 100 \mathrm{U} / \mathrm{ml}$ penicillin and $100 \mathrm{mg} / \mathrm{ml}$ streptomycin, in addition to $5 \times 10^{2}$ of the transfected $786-\mathrm{O}$ cells. Plates were incubated at $37.8^{\circ} \mathrm{C}$ with $5 \% \mathrm{CO}_{2}$ in a humidified incubator. Images of the plates were captured on day 14, and the number of colonies was quantified using Quantity One software (version 4.0.3; Bio-Rad Laboratories, Inc., Hercules, CA, USA). The assays were performed 3 times. The data are presented as mean average.

Cell invasion assays. Cell invasion assays were performed using a Transwell chamber (BD Biosciences, Franklin Lakes, NJ, USA). Transfected NC siRNA cells were used as a control group. The assays were performed 3 times. In the invasion assay, $2 \times 10^{4}$ transfected cells in serum-free medium was seeded into the top chamber, which was pre-coated with Matrigel (BD Biosciences). Following incubation for $24 \mathrm{~h}$ at $37^{\circ} \mathrm{C}$, the membranes were fixed using methyl alcohol $(100 \%)$ and then stained with $0.1 \%$ crystal violet. The number of cells that passed through the membranes were counted under a light microscope.

Statistical analysis. SPSS software (version 13.0; SPSS, Inc., Chicago, IL, USA) was employed for the analysis of all data. Data are expressed as the mean \pm standard deviation. A Student's t-test were used to determine the significance of the differences between groups. $\mathrm{P}<0.05$ was considered to indicate a statistically significant difference.

\section{Results}

Silencing of miR-21 inhibits tumor growth in the mouse renal cancer model. To investigate whether the miR-21 inhibitor inhibited tumor growth, the weight of tumors were measured following miR-21 inhibitor treatment. Fig. 1A presents images of the tumors from the nude mice models in the three groups (NC, miR-21 mimic and miR-21 inhibitor). Compared with the $\mathrm{NC}$, the weight of the tumors in the miR-21 inhibitor group was significantly decreased $(\mathrm{P}<0.05$; Fig. 1B). The weight of the tumors in the miR-21 mimic group was significantly increased compared with the $\mathrm{NC}(\mathrm{P}<0.05$; Fig. 1B).

\section{Downregulation of miR-21 expression increases PDCD4} expression in the mouse renal cancer model. To evaluate the association between PDCD4 and miR-21 in the nude mouse RCC models, whether the transplantation of 786-O cells with miR-21 mimic or miR-21 inhibitor affected the expression of PDCD4 was determined. Downregulation of endogenous miR-21 by the miR-21 inhibitor resulted in a significant increase in the expression of PDCD4 protein compared with the NC group ( $\mathrm{P}<0.05$; Fig. $1 \mathrm{C}$ and $1 \mathrm{D})$. By contrast, there was a significant decrease in the levels of PDCD4 protein in the miR-21 mimic group compared with the NC group $(\mathrm{P}<0.05$; Fig. 1C and D). The expression of was significantly upregulated in the miR-21 mimic group compared with the NC group, whilst it was significantly reduced in the miR-21 inhibitor group $(\mathrm{P}<0.05$; Fig. 1E). However, there was no significant difference in the expression of PDCD4 mRNA between the three groups $(\mathrm{P}>0.05$; Fig. $1 \mathrm{~F})$.

Immunohistochemical analysis of PDCD4 was performed (Fig. 2), indicating that PDCD4 was localized to the cytoplasm. 
A

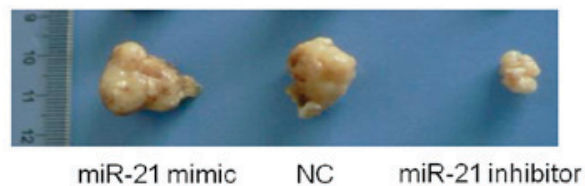

C

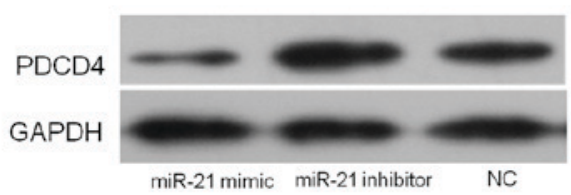

E

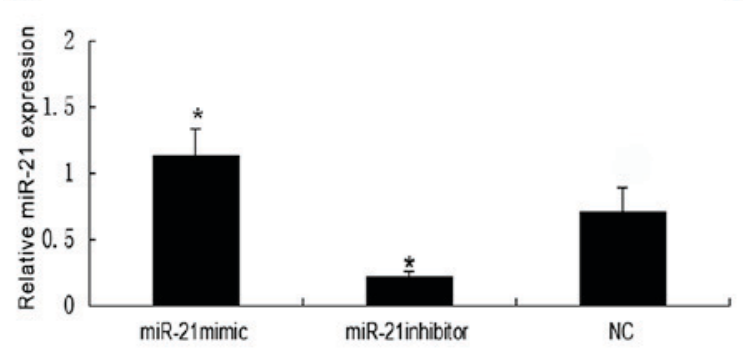

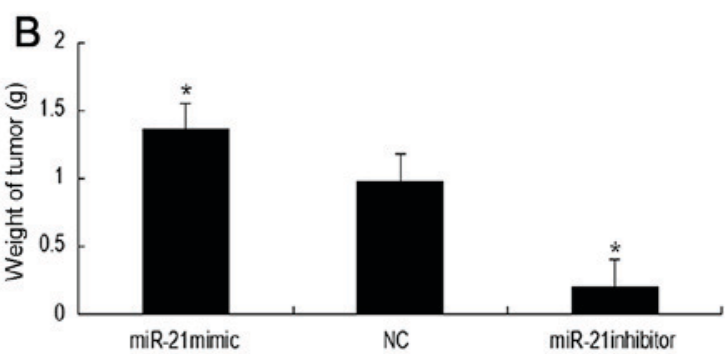

D
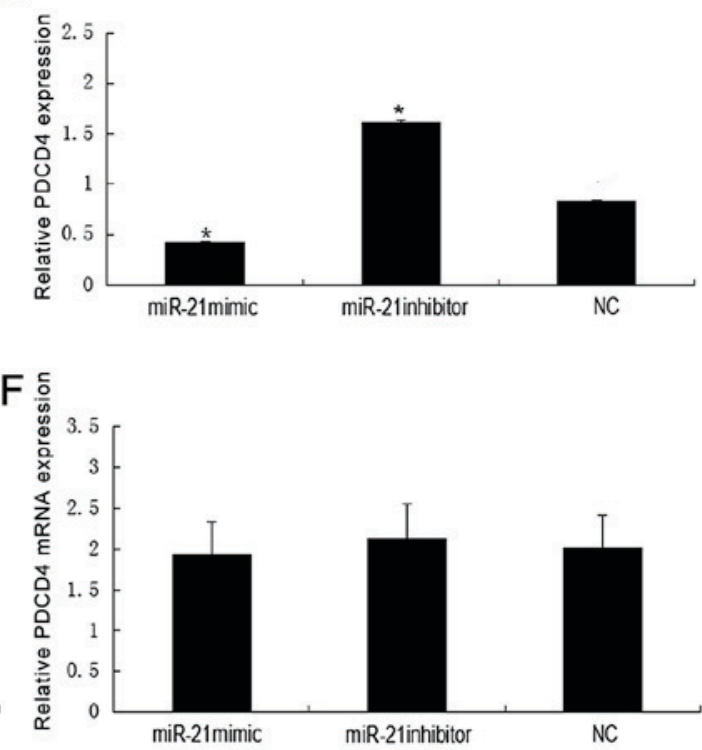

Figure 1. Downregulation of miR-21 expression increases PDCD4 expression in vivo. (A) Images of tumors from the nude mouse models in the NC, miR-21 mimic and miR-21 inhibitor groups. (B) The weight of tumors in the miR-21 mimic group was significantly increased compared with the NC group, and the weight of tumors in the miR-21 inhibitor group was significantly decreased compared with the NC group. Western blotting for PDCD4 (C) image and (D) densitometric analysis relative to GAPDH. (E) Relative expression of miR-21 determined by stem-loop RT-qPCR. (F) Relative expression of PDCD4 as determined by RT-qPCR. Student's t-test was used for comparisons of two independent groups. " P<0.05 vs. the NC group. miR, microRNA; NC, negative control; PDCD4, programmed cell death 4; RT-qPCR, reverse transcription-quantitative polymerase chain reaction.
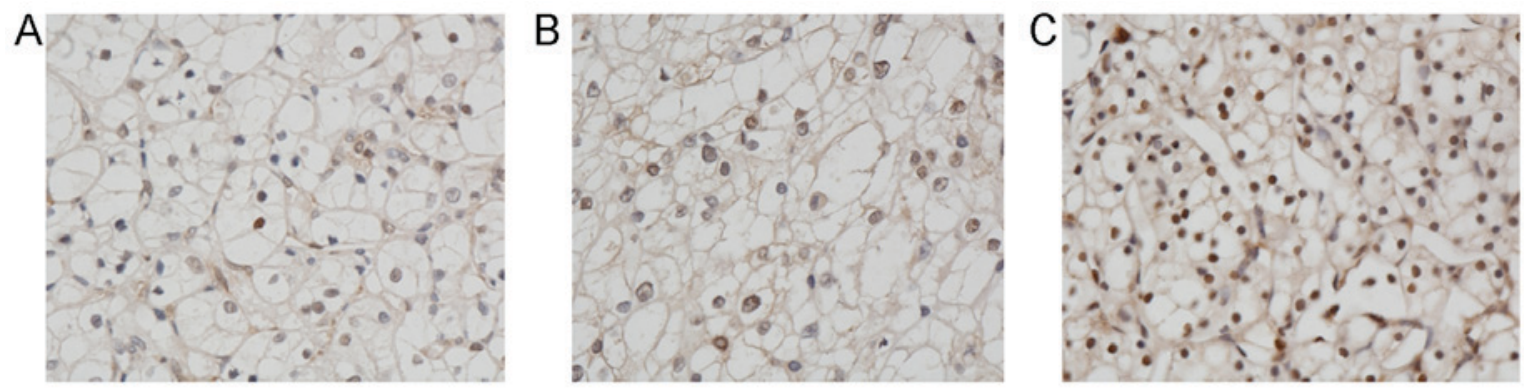

Figure 2. Immunostaining of PDCD4 protein in tumor tissues from the nude mouse models. (A) miR-21 mimic group. (B) NC group. (C) miR-21 inhibitor group. Magnification, x400. PDCD4, programmed cell death 4; NC, negative control; miR, microRNA.

Reduced or complete loss of PDCD4 expression was detected in the miR-21 mimic group (Fig. 2A). The immunocytochemical staining depth was moderate in the NC group (Fig. 2B). By contrast, strong immunopositivity for PDCD4 was observed in the miR-21 inhibitor group (Fig. 2C).

Silencing of PDCD4 induces tumor cell proliferation, colony formation, migration and invasion. The effect of silencing PDCD4 on cell proliferation was investigated by transfecting PDCD4 siRNA and control siRNA into 786-O cells. To examine whether there was a change in the number of proliferating 786-O cells following transfection, the cells were labeled with EdU to measure active DNA synthesis and Hoechst 33342 to illustrate the nuclei of all cells (Fig. 3A). It was identified that silencing of PDCD4 significantly promoted cell proliferation. According to the results of fluorescent microscopic analysis, the mean percentage of newly formed cells that incorporated EdU was $28.6 \%$ in the NC siRNA group and $44.7 \%$ in the PDCD4 siRNA-transfected cells $(\mathrm{P}<0.05$; Fig. 3B). 

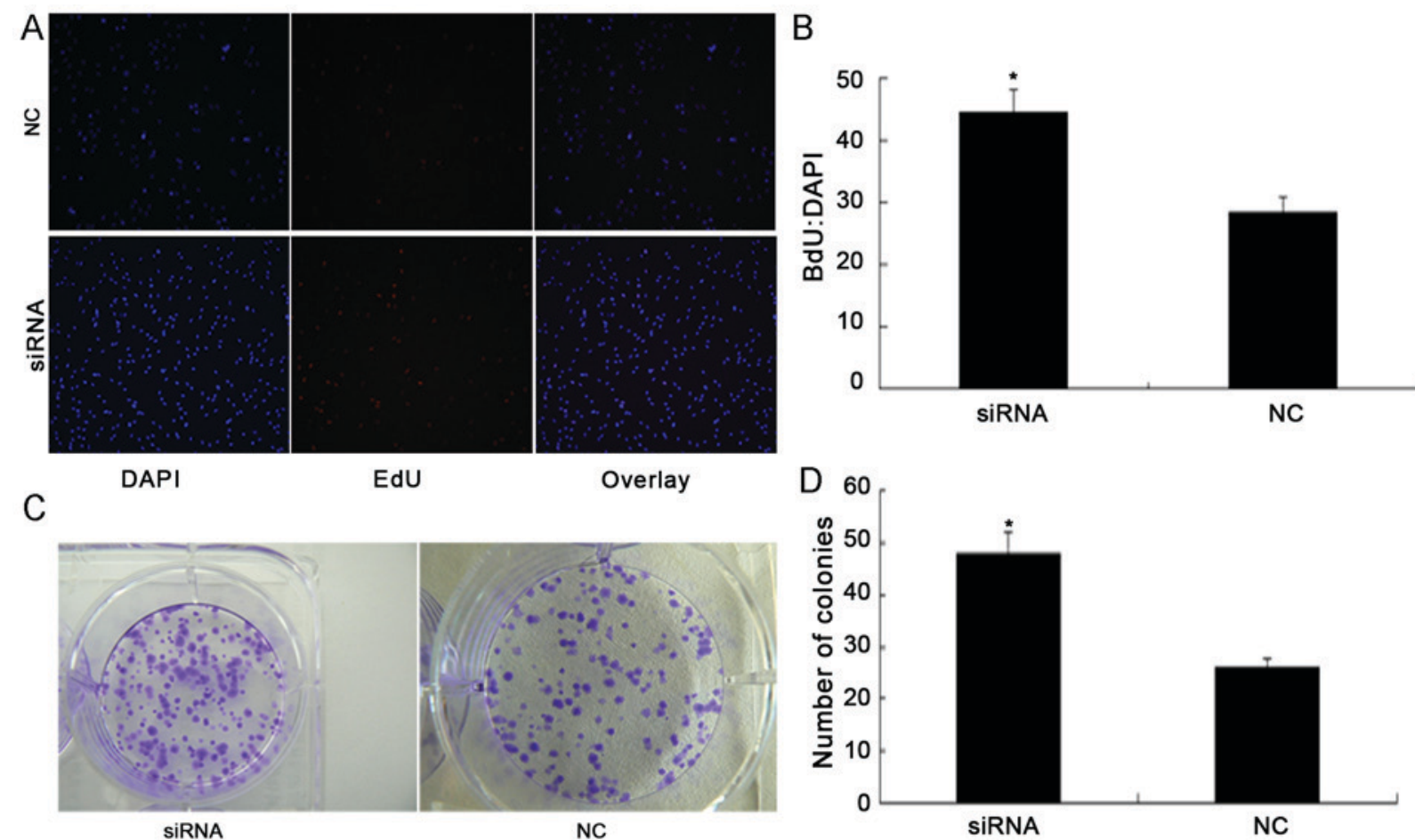

Figure 3. PDCD4 knockdown promotes renal cancer cell proliferation. (A) EdU proliferation assay. Proliferating cells that have incorporated EdU are stained red, while the nuclei of all cells is stained blue with Hoechst 33342. All experiments were repeated three times. (B) Proliferative ability of 786-O cells following transfection with PDCD4 siRNA and NC siRNA. (C) Images of colony formation assay of 786-O cells transfected with NC siRNA and PDCD4 siRNA captured on day 14. (D) The colony-forming ability of 786-O cells was significantly increased in the PDCD4 siRNA-transfected group compared with the NC group 14 days after transcription. Student's t-test was used for comparisons of two independent groups. "P<0.05 vs. the NC group. PDCD4, programmed cell death 4; NC, negative control; siRNA, small interfering RNA.

A colony formation assay was performed to determine whether PDCD4 knockdown promoted the colony forming ability of 786-O cells (Fig. 3C). The colony formation assay demonstrated that the total number of colonies in the PDCD4 siRNA-transfected group was significantly increased compared with the $\mathrm{NC}$ group $(\mathrm{P}<0.05$; Fig. 3D). Furthermore, the results of the invasion assay demonstrated that silencing the expression of PDCD4 significantly increased invasion of 786-O cells compared with the $\mathrm{NC}$ group $(\mathrm{P}<0.05$; Fig. $4 \mathrm{~A}$ and $\mathrm{B})$.

Silencing of PDCD4 induces tumor growth in the mouse renal cancer model. To investigate whether PDCD4 silencing induces tumor growth, tumor weight was measured following PDCD4 siRNA and NC treatment. Fig. 4C illustrates the tumors from the nude mouse models. Compared with the NC group, tumor weight in the PDCD4 siRNA group was significantly increased ( $\mathrm{P}<0.05$; Fig. 4D).

\section{Discussion}

To the best of our knowledge, the present study is the first to demonstrate that PDCD4 is negatively regulated by miR-21, in addition to being able to suppress tumor growth and metastasis in a nude mouse renal cancer model. In a previous study, it was revealed that there is a conserved target site for miR-21 within the PDCD4 3' untranslated region at nucleotides 228-249 (11). It has also been reported that miR-21 is able to regulate the Ras/mitogen-activated protein kinase kinase/extracellular signal-regulated kinase signaling pathway and therefore affect tumor formation (31). Additionally, a meta-analysis has indicated that miR-21 is able to act as an important biomarker for the prognosis of various types of cancer (32). Xu et al (33) demonstrated that the downregulation of miR-21 increased the sensitivity of lung cancer cells to cisplatin in vitro and in vivo. Wang et al (34) reported that miR-21 expression was significantly increased in hepatocellular carcinoma tissues compared with normal adjacent liver tissues. Furthermore, Li et al (28) demonstrated that the tumor suppressor PDCD4 was negatively regulated at a post-transcriptional level by miR-21, and that miR-21 induced cell proliferation and invasion/metastasis in RCC. PDCD4 expression is also significantly positively associated with RCC metastasis, and tumor stage and grade (35).

To further investigate the association between PDCD4 and miR-21 in vivo, BALB/c male nude mice and 786-O cells were used in the present study to establish a nude mouse renal cancer model. It was identified that tumor weight in the miR-21 inhibitor group was significantly decreased compared with the NC group. Compared with the NC group, tumor weight in the miR-21 mimic group was significantly increased. Subsequently, the expression of PDCD4 in the NC group, miR-21 inhibitor group and miR-21 mimic group was analyzed by western blotting. There was loss or reduced expression of PDCD4 protein in the miR-21 mimic group; however, PDCD4 protein was highly expressed in the miR-21 inhibitor group compared with the NC group. Similar results were revealed by immunohistochemistry. In addition, there was no significant difference between PDCD4 mRNA levels between the 
A

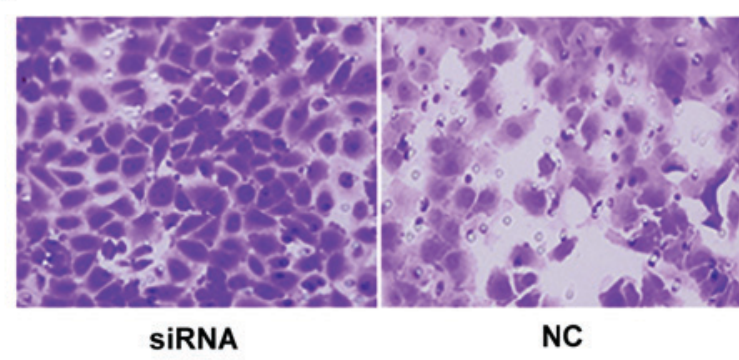

$\mathrm{C}$

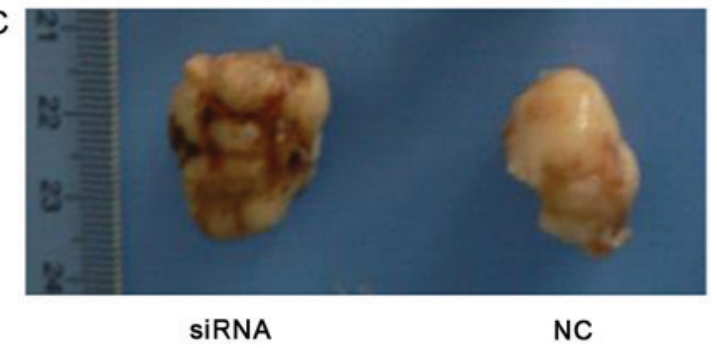

B
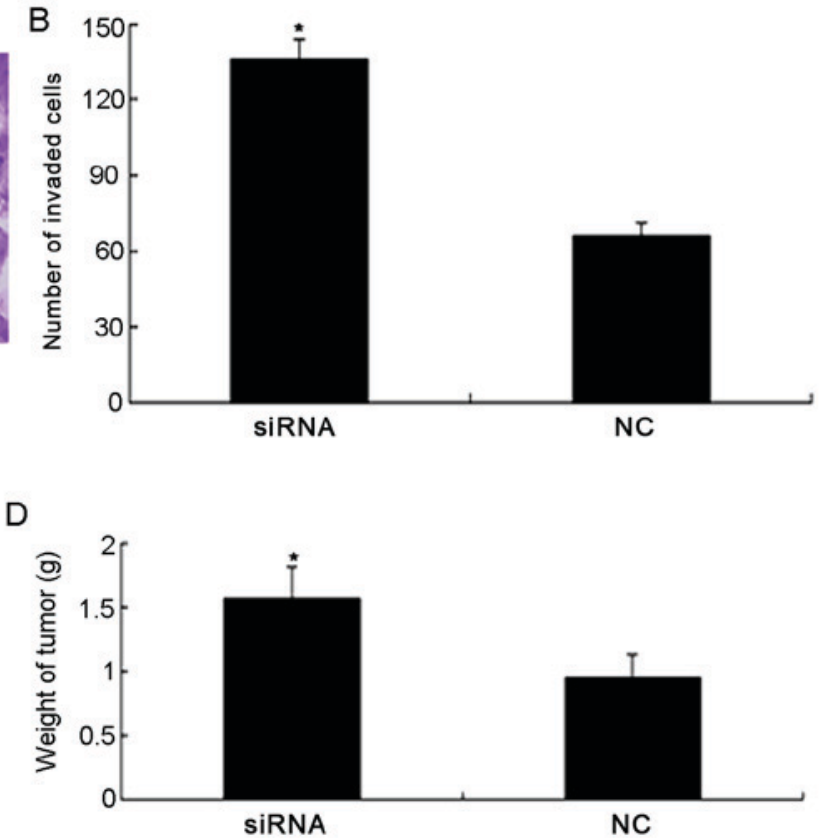

Figure 4. (A) Representative images of Transwell invasion assay. The 786-O cells were transfected with NC siRNA and PDCD4 siRNA. (B) The ability of cells to invade in the PDCD4 siRNA group was significantly increased compared with the NC group. (C) Image of tumors from the nude mice NC and PDCD4 siRNA groups. (D) The weight of tumors in the PDCD4 siRNA group were significantly increased compared with the NC. Student's t-test was used for comparisons of two independent groups. ${ }^{\mathrm{P}}<0.05$ vs. the $\mathrm{NC}$ group.

three groups (miR-21 mimic, miR-21 inhibitor and negative control). The results of the present study, which were similar to the previously published study of human renal cancer tissue and cell lines (28), demonstrated that miR-21 downregulates PDCD4 at a post-transcriptional level, and promotes cell colony formation and proliferation in the nude mouse renal cancer model.

PDCD4 has been known to be a tumor suppressor gene and potential target for anticancer therapies for several years $(11,14,16-21)$. Reduced PDCD4 expression has been reported in $>5$ types of human tumors, including those of the lung, brain, breast, colon and pancreas (11). In colon cancer cell lines, Wang et al (36) has demonstrated that downregulation of PDCD4 leads to an increase in colon carcinoma cell invasion. Furthermore, the knockdown of PDCD4 in the study by Wang et al (36) was associated with a significant reduction in E-cadherin expression and accumulation of active $\beta$-catenin in the nucleus of these cells. In the same series of experiments, PDCD4 knockdown resulted in an increase in activator protein (AP)-1-dependent transcription. These results indicate that reduced PDCD4 expression promotes cancer cell invasion, and that this is associated with the activation of $\beta$-catenin, E-cadherin and AP-1-dependent transcription. Another study demonstrated that decreased PDCD4 expression was significantly associated with the clinical stage of adenoid cystic carcinoma (37). Li et al (35) observed that decreased PDCD4 expression was significantly positively associated with metastasis, and tumor stage and grade in RCC.

In the present study, the effect of PDCD4 on miR-21 expression was investigated. PDCD4 siRNA was transfected into 786-O cells, and PDCD4 mRNA and protein levels were found to be significantly decreased compared with the NC group. Silencing PDCD4 also significantly promoted 786-O cell proliferation, migration and invasion, similar to the effects observed after miR-21 overexpression.

In conclusion, the results of the present study and previous studies indicate that PDCD4 and miR-21 serve important roles in RCC. The results also suggest that the promotion of PDCD4 expression or inhibition of miR-21 expression may constitute effective novel therapeutic strategies for the treatment of renal cancer.

\section{Acknowledgements}

The present study was supported by the Henan Provincial Science and Technology Plan Foundation (grant no. B2014225013).

\section{References}

1. van Spronsen DJ, Mulders PF and De Mulder PH: Novel treatments for metastatic renal cell carcinoma. Crit Rev Oncol Hematol 55: 177-191, 2005.

2. Cohen HT and McGovern FJ: Renal-cell carcinoma. N Engl J Med 353: 2477-2490, 2005.

3. Hammond SM: MicroRNAs as oncogenes. Curr Opin Genet Dev 16: 4-9, 2006.

4. Esquela-Kerscher A and Slack FJ: Oncomirs-microRNAs with a role in cancer. Nat Rev Cancer 6: 259-269, 2006.

5. Calin GA, Dumitru CD, Shimizu M, Bichi R, Zupo S, Noch E, Aldler H, Rattan S, Keating M, Rai K, et al: Frequent deletions and down-regulation of micro- RNA genes miR15 and miR16 at 13q14 in chronic lymphocytic leukemia. Proc Natl Acad Sci USA 99: 15524-15529, 2002.

6. Chan JA, Krichevsky AM and Kosik KS: MicroRNA-21 is an antiapoptotic factor in human glioblastoma cells. Cancer Res 65: 6029-6033, 2005.

7. Lu J, Getz G, Miska EA, Alvarez-Saavedra E, Lamb J, Peck D, Sweet-Cordero A, Ebert BL, Mak RH, Ferrando AA, et al: MicroRNA expression profiles classify human cancers. Nature 435: 834-838, 2005. 
8. Meng F, Henson R, Lang M, Wehbe H, Maheshwari S, Mendell JT, Jiang J, Schmittgen TD and Patel T: Involvement of human micro-RNA in growth and response to chemotherapy in human cholangiocarcinoma cell lines. Gastroenterology 130: 2113-2129, 2006.

9. Roldo C, Missiaglia E, Hagan JP, Falconi M, Capelli P, Bersani S, Calin GA, Volinia S, Liu CG, Scarpa A and Croce CM MicroRNA expression abnormalities in pancreatic endocrine and acinar tumors are associated with distinctive pathologic features and clinical behavior. J Clin Oncol 24: 4677-4684, 2006

10. Si ML, Zhu S, Wu H, Lu Z, Wu F and Mo YY: miR-21-mediated tumor growth. Oncogene 26: 2799-2803, 2007.

11. Asangani IA, Rasheed SA, Nikolova DA, Leupold JH, Colburn NH, Post S and Allgayer H: MicroRNA-21 (miR-21) post-transcriptionally downregulates tumor suppressor Pdcd 4 and stimulates invasion, intravasation and metastasis in colorectal cancer. Oncogene 27: 2128-2136, 2008.

12. Frankel LB, Christoffersen NR, Jacobsen A, Lindow M, Krogh A and Lund AH: Programmed cell death 4 (PDCD4) is an important functional target of the microRNA miR-21 in breast cancer cells. J Biol Chem 283: 1026-1033, 2008.

13. Zhu S, Wu H, Wu F, Nie D, Sheng S and Mo YY: MicroRNA-21 targets tumor suppressor genes in invasion and metastasis. Cell Res 18: 350-359, 2008.

14. Cmarik JL, Min H, Hegamyer G, Zhan S, Kulesz-Martin M, Yoshinaga H, Matsuhashi S and Colburn NH: Differentially expressed protein Pdcd4 inhibits tumor promoter-induced neoplastic transformation. Proc Natl Acad Sci USA 96: 14037-14042, 1999.

15. Lee S, Bang S, Song K and Lee I: Differential expression in normal-adenoma-carcinoma sequence suggests complex molecular carcinogenesis in colon. Oncol Rep 16: 747-754, 2006.

16. Mudduluru G, Medved F, Grobholz R, Jost C, Gruber A Leupold JH, Post S, Jansen A, Colburn NH and Allgayer H: Loss of programmed cell death 4 expression marks adenoma-carcinoma transition, correlates inversely with phosphorylated protein kinase $\mathrm{B}$, and is an independent prognostic factor in resected colorectal cancer. Cancer 110: 1697-1707, 2007.

17. Yang HS, Jansen AP, Nair R, Shibahara K, Verma AK, Cmarik JL and Colburn NH: A novel transformation suppressor, Pdcd4, inhibits AP-1 transactivation but not NF-kappaB or ODC transactivation. Oncogene 20: 669-676, 2001.

18. Yang HS, Jansen AP, Komar AA, Zheng X, Merrick WC, Costes S, Lockett SJ, Sonenberg N and Colburn NH: The transformation suppressor Pdcd4 is a novel eukaryotic translation initiation factor 4A binding protein that inhibits translation. Mol Cell Biol 23: 26-37, 2003.

19. Jansen AP, Camalier CE and Colburn NH: Epidermal expression of the translation inhibitor programmed cell death 4 suppresses tumorigenesis. Cancer Res 65: 6034-6041, 2005.

20. Hilliard A, Hilliard B, Zheng SJ, Sun H, Miwa T, Song W, Göke R and Chen YH: Translational regulation of autoimmune inflammation and lymphoma genesis by programmed cell death 4. J Immunol 177: 8095-8102, 2006.

21. LaRonde-LeBlanc N, Santhanam AN, Baker AR, Wlodawer A and Colburn NH: Structural basis for inhibition of translation by the tumor suppressor Pdcd4. Mol Cell Biol 27: 147-156, 2007.

22. Baffa R, Fassan M, Volinia S, O'Hara B, Liu CG, Palazzo JP, Gardiman M, Rugge M, Gomella LG, Croce CM and Rosenberg A: MicroRNA expression profiling of human metastatic cancers identifies cancer gene targets. J Pathol 219: 214-221, 2009.
23. Selaru FM, Olaru AV, Kan T, David S, Cheng Y, Mori Y, Yang J, Paun B, Jin Z, Agarwal R, et al: MicroRNA-21 is overexpressed in human cholangiocarcinoma and regulates programmed cell death 4 and tissue inhibitor of metalloproteinase 3. Hepatology 49: 1595-1601, 2009.

24. Lu Z, Liu M, Stribinskis V, Klinge CM, Ramos KS, Colburn NH and Li Y: MicroRNA-21 promotes cell transformation by targeting the programmed cell death 4 gene. Oncogene 27: 4373-4379, 2008.

25. Zhou L, Yang ZX, Song WJ, Li QJ, Yang F, Wang DS, Zhang N and Dou KF: MicroRNA-21 regulates the migration and invasion of a stem-like population in hepatocellular carcinoma. Int $\mathrm{J}$ Oncol 43: 661-669, 2013.

26. Chen Y, Liu W, Chao T, Zhang Y, Yan X, Gong Y, Qiang B, Yuan J, Sun M and Peng X: MicroRNA-21 down-regulates the expression of tumor suppressor PDCD4 in human glioblastoma cell T98G. Cancer Lett 272: 197-205, 2008.

27. Hiyoshi Y, Kamohara H, Karashima R, Sato N, Imamura Y, Nagai Y, Yoshida N, Toyama E, Hayashi N, Watanabe M and Baba H: MicroRNA-21 regulates the proliferation and invasion in esophageal squamous cell carcinoma. Clin Cancer Res 15: 1915-1922, 2009.

28. Li X, Xin S, He Z, Che X, Wang J, Xiao X, Chen J and Song X: MicroRNA-21 (miR-21) post-transcriptionally downregulates tumor suppressor PDCD4 and promotes cell transformation, proliferation, and metastasis in renal cell carcinoma. Cell Physiol Biochem 33: 1631-1642, 2014.

29. Care and Use of Laboratory Animals of the National Institutes of Health.8th edition. Washington (DC): National Academies Press (US), 2011.

30. Livak KJ and Schmittgen TD: Analysis of relative gene expression data using real-time quantitative PCR and the 2(-Delta Delta C(T)) method. Methods 25: 402-408, 2001.

31. Hatley ME, Patrick DM, Garcia MR, Richardson JA, Bassel-Duby R, van Rooij E and Olson EN: Modulation of K-Ras-dependent lung tumorigenesis by MicroRNA-21. Cancer Cell 18: 282-293, 2010.

32. Zhou X, Wang X, Huang Z, Wang J, Zhu W, Shu Y and Liu P: Prognostic value of miR-21 in various cancers: An updating meta-analysis. PLoS One 9: e102413, 2014.

33. Xu L, Huang Y, Chen D, He J, Zhu W, Zhang Y and Liu X: Downregulation of miR-21 increases cisplatin sensitivity of non-small-cell lung cancer. Cancer Genet 207: 214-220, 2014.

34. Wang WY, Zhang HF, Wang L, Ma YP, Gao F, Zhang SJ and Wang LC: miR-21 expression predicts prognosis in hepatocellular carcinoma. Clin Res Hepatol Gastroenterol 38: 715-719, 2014.

35. Li X, Xin S, Yang D, Li X, He Z, Che X, Wang J, Chen F, Wang $X$ and Song X: Down-regulation of PDCD4 expression is an independent predictor of poor prognosis in human renal cell carcinoma patients. J Cancer Res Clin Oncol 138: 529-535, 2012.

36. Wang Q, Sun Z and Yang HS: Downregulation of tumor suppressor Pdcd4 promotes invasion and activates both beta-catenin/Tcf and AP-1-dependent transcription in colon carcinoma cells. Oncogene 27: 1527-1535, 2008.

37. Qi C, Shao Y, Li N, Zhang C, Zhao M and Gao F: Prognostic significance of PDCD4 expression in human salivary adenoid cystic carcinoma. Med Oncol 30: 491, 2013. 